\title{
Manufacturing knowledge management strategy
}

- Purpose: To understand the components of knowledge management strategy from the perspective of staff in UK manufacturing organisations.

- Methodology/Approach: Empirical approach - data collected during workshops conducted in two manufacturing organisations.

- Findings: The key components of a knowledge management strategy, and the relationships between it and manufacturing strategy and corporate strategy. Other findings include: the nature of knowledge in manufacturing organisations; the relevance of (in)formal processes; top-down and bottom-up communication; taking ownership for information processes.

- Research limitations/implications: Implications for theory development in manufacturing strategy and its alignment with corporate strategy.

- Practical implications: Implications for doing knowledge management, and developing strategy, in manufacturing organisations.

- Originality/value of paper: Investigates knowledge management strategy in the light of manufacturing strategy and corporate strategy.

Research paper; Case study paper

Keywords: Manufacturing strategy, operations, knowledge management, knowledge management strategy, group decision support.

\section{Introduction}

The focus of the research reported here is on knowledge management strategy. We explore what issues are important to representatives from two manufacturing organisations when designing action plans for better knowledge management in their organisation. One intention of the research was to establish what knowledge the representatives thought might benefit from being managed. Another was to examine how knowledge management strategy links to other aspects of strategy. The manufacturing literature (Hill, 1987; Misterek et al., 1992) typically distinguishes between manufacturing strategy and corporate strategy. This distinction makes it easier to discuss the relationship to knowledge management strategy than for non-manufacturing organisations. Hence this paper concentrates on the two manufacturing organisations out of a larger group that were originally studied.

The gap in the literature which this paper addresses is illustrated by Bhatt [, 2000 \#974] who observes "a few studies have addressed knowledge strategies briefly, but these studies, at best, 
can be described as personal viewpoints and opinions as they lack theoretical rigor and practical applications”. This paper concentrates on practice, but aims to shed light on relevant theory. We provide insight from the developers and implementers of strategy as to the content of, and rationale behind, their knowledge management strategy. This strategy needed to encompass the delivery of different types of knowledge (formal or informal) in different formats (e.g. written, oral, electronic), involving different influences to support their owners' co-operation. It also needed to address operations, manufacturing strategy and corporate strategy.

There are many ways of looking at knowledge management in abstract. This paper adopts a process viewpoint. The term "process" is often used in two different ways in the manufacturing strategy literature. One is for "a pattern or procedure in which manufacturing strategy is developed and implemented" (Minor et al., 1994), by contrast with the content of that strategy. The other is the process of manufacturing the product itself. The process viewpoint adopted here embraces both of these meanings, and goes further, to cover all the business processes in the organisation.

The theory of business processes distinguishes between core, support and management processes (Earl, 1994). The core processes are defined as the ones that make the organisation what it is; the ones performed directly for the external customers whom the organisation exists to serve. For a manufacturing organisation, the manufacturing process - often called manufacturing operations - will certainly be one of the core processes. Support processes are typically for internal customers, and often "administrative” in nature. Management processes, such as strategy and planning, direct and underpin the core and support processes. Thus the manufacturing strategy process as defined by Minor et al is the management process that corresponds to the core manufacturing process. This view is now becoming part of mainstream management thinking. For example, the latest version of the ISO9000 family of standards for Quality Management Systems also takes a process viewpoint, the ISO9000 term realisation process being equivalent to Earl's core process.

Assuming that the knowledge in an organisation is linked to its business processes, it should to be helpful to make a similar distinction about the knowledge requirements (see Edwards and Kidd (2003a) for a further discussion). By definition, core processes need to be done as well as possible, since they actually produce the outputs for the external customer. Any improvement in, or addition to the organisation's core knowledge - the knowledge needed to perform the core processes - should therefore produce a corresponding improvement in the organisation’s overall performance. By contrast, support processes only need to be done “well 
enough". Thus beyond a certain point, improvements in or additions to the organisation's support knowledge will have little or no effect. The importance of management knowledge will correspondingly depend on whether it relates to the management of a core or support process.

The structure of the paper is as follows. We first position our work within the literature on managing knowledge in manufacturing organisations. Next, we describe our research approach, concentrating on the use of small group workshops for data capture. Then we present the findings from two organisations and, finally, we discuss the transferable results from this study.

\section{Categorising knowledge in manufacturing organisations}

The manufacturing strategy literature provides a useful foundation for categorising knowledge in manufacturing organisations. Misterek et al (1992) clearly distinguish manufacturing strategy from corporate strategy, the latter covering marketing and finance, amongst other topics. Hayes and Wheelwright (1984) identified four stages of progression in manufacturing strategy, from internally neutral to externally supportive, during which the relationship between manufacturing strategy and corporate strategy changes. Hill (1987) divided manufacturing strategy into two components, structure and infrastructure. Structure covers process and technology - the design, development and implementation of manufacturing strategy. Infrastructure refers to longer term aspects - human resource policies, quality systems, organisational culture, IT. The infrastructure supports the structure.

Combining these aspects, a suitable set of top level categories for the content of the knowledge in a manufacturing organisation seems to be: manufacturing operations, manufacturing strategy (subdivided into structure and infrastructure), and corporate strategy.

Other 'dimensions' that will also be used in the later discussion are the division between core and support processes explained earlier, and also the difference between formal and informal mechanisms. An important linkage between the different dimensions is that whilst the "structure” part of manufacturing strategy is by definition almost entirely formal, a crucial informal mechanism, organisational culture, is part of Hill's infrastructure.

\section{Managing knowledge in manufacturing organisations}

There is a wealth of research which explains how manufacturing organisations can improve knowledge management. Applied research on the management of knowledge in manufacturing organisations has explored the link between knowledge management and: 
organisational performance (Germain et al., 2001); information technology (Beckett et al., 2000; Prasad, 2000; Leseure and Brookes, 2001); competitiveness (Paiva et al., 2002); the transfer of best practice (Collinson, 1999); inter-organisational networking (Robertson et al., 1996; Chaston and Mangles, 2000); organisational learning (Mohanty and Deshmukh, 1999; Chaston et al., 2001; Ordonez de Pablos, 2002; Robinson et al., 2003).

However, from the above list only two papers are closely related with our work: those of Collinson (1999) and Mohanty and Deshmukh (1999). Collinson examined the transfer of best practice between two manufacturing organisations - the aim of the companies was to improve their manufacturing processes. He describes the changes to working practices made in the recipient company. These include: process control; quality monitoring; process enhancement groups; cross-functional working; improved communication; a customer focus. These changes contributed to improvements in defects rate and yield. As may be seen, these covered both the core manufacturing process and its related management process. While his study has important similarities with ours (we are all interested in improving manufacturing processes), his study differs from ours in many ways, most importantly that his focus was the transfer of best practice between two companies. Our focus is the development of knowledge management strategy by the staff in two companies, where often they had no best practice to transfer and they have to generate practice from new.

Mohanty and Deshmukh (1999) evaluate manufacturing strategy for the selection and evaluation of advanced manufacturing technology, their focus being organisation learning. They suggest a "group-based participatory evaluation process ... to provide a prioritised list of strategic initiatives”, page 314-5. They use group decision support approaches to facilitate the evaluation of strategic alternatives by a group of decision-makers. Their study differs from ours in that their focus is on the evaluation of strategic alternatives for manufacturing strategy, while ours is on the design and negotiation of strategic action plans for knowledge management strategy.

The paper by Mohanty and Deshmukh (1999) also helps to confirm the suitability of group working for exploring aspects of strategy in manufacturing organisations. Perhaps the most widely-known example of this kind is the work of Platts and Gregory [, 1992 \#4156]. They devised a process for developing manufacturing strategy, several of whose stages rely heavily on facilitated participative workshops. Nevertheless, we are not aware of any previous work on knowledge management strategy in manufacturing organisations that has taken a workshop approach. 
We will now introduce our research methodology, including the knowledge management workshops, in preparation for reviewing their results.

\section{Research methodology}

The overall research methodology used in this study was as set out below. Note that the term "client" refers to the main contact in that organisation. For each organisation, the steps proceeded as follows:

- Design and agreement of workshop agenda to address the organisation's concerns (with the client and then the participants).

- $\quad$ The workshop.

- Oral de-briefing of client.

- Post-workshop data analysis (of maps, exit questionnaires, researcher observations).

- Written report to client and participants.

- Client/participant feedback on report.

- Identification of generic and specific themes.

\section{Workshop format}

Both workshops were an entire day in length for a single organisation. We will refer to the two organisations as "HighTechManuf" and "ManufIndProd" to preserve their anonymity. We suggested there should be 6-10 organisational members in the workshop: too few participants, and the range of views represented would be narrow; too many participants, and the group dynamics become more complex for the facilitator. In the event, 7 attended from HighTechManuf and 10 from ManufIndProd.

The workshops were conducted according to an approach named Journey Making (Eden and Ackermann, 1998). To build commitment to an action plan, JOURNEY Making suggests that

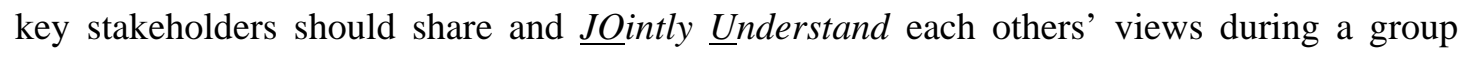
workshop. They should $\underline{R} e f l e c t$ on the diverse nature of those views and $\underline{N E g o t i a t e}$ a strateg $\underline{Y}$ which all the stakeholders want (in our case) to improve knowledge management.

The workshops used a facilitated computer-supported group decision support system. Pairs of participants shared a laptop computer which was running a brainstorming software package and was networked to the facilitator's computer. To share their views on knowledge management in their organisation, participants typed them into their laptop (using only a few words to indicate the view - so views could be understandable to others). The view would be transmitted to the facilitator's computer which was linked to a projector. There the facilitator loosely clustered the views by content to position similar views beside one another and 
thereby help the participants to absorb the views. After participants finished sharing all their views, all the clusters were projected onto a screen in map form (see Figures 1 and 2). Participants then read the map of clusters and shared more views as they were stimulated by what they read. This would normally result in a total of up to 100 different views being displayed.

To more fully understand the relationships between the views, group discussion around the clusters would ensue. To graphically represent a relationship on the map, a link would be inserted between appropriate views in the form of an arrow, for example, knowledge of “customer's own experiences” provides "customer feedback" of "what the customer wants" (in the top right of Figure 2). The participants, as a group, could then amend or move the views and the clusters. As well as linking and clustering views, everything else about the entire map could be amended until the participants were agreed that it represented their position on the issues.

The advantages of computer support in this context include speed, anonymity, creativity and accuracy. They are discussed in detail in Shaw (2003).

\section{A flexible agenda for the workshops}

It is generally accepted that knowledge management must vary between organisations. This implies that the participants must design their own agenda. However, as the organisations were relatively new to knowledge management, a starting-point was needed. We therefore initially suggested the following four sessions:

- Session 1: "What knowledge informs our business?"

- Session 2: "What knowledge management processes currently exist in our organisation?”

- Session 3: "What knowledge management processes should exist in our organisation?”

- Session 4: "How do we (or should we) evaluate how good these processes actually are?”

In both workshops the groups partially followed this agenda (through Sessions 1 and 2), and then decided to design their own sessions before returning to this agenda (as discussed below). Each session produces at least one map, but to save space we have included only one map from Session 1 of each workshop. 


\section{The organisations and the findings}

\section{HighTechManuf}

HighTechManuf is a high technology manufacturing group, privately owned with $£ 100 \mathrm{M}$ turnover and 800 employees. It had recently been formed through a merger of four constituent companies, two of which had been competitors. There had been little pursuit of knowledge management previously, but this was now seen as important to "start acting like a large company as now we cannot act like a small informal company". The client (who was tasked with improving the organisation's processes) saw that improving knowledge management practices was an important move - to get knowledge flowing between the former constituent companies.

Most of the 7 workshop participants were engineers by background. The participants thought that knowledge management was important to HighTechManuf, evident through their agreement with the statement on their exit questionnaire that "I think that knowledge management is an important issue in HighTechManuf” (average 1.57, where $1=$ Strongly Agree and 5= Strongly Disagree).

The first session asked "What knowledge informs HighTechManuf?" and produced the map shown in Figure 1. The participants identified 11 clusters, ranging from " $\mathrm{R} \& \mathrm{D}$ " to "customer requirements". Note that we have retained the exact terms used by the participants, even when they include errors of spelling or grammar, as with the cluster named "metallurgically capability".

\{Take in Figure 1: “A map from the HighTechManuf workshop”\}

There was also discussion about the currency of knowledge, around the statement "if it is not owned and updated, is it of any value". It was agreed that ideally all knowledge and information should be up-to-date, and that processes based on ownership should be implemented to ensure this.

Reflecting on the range of knowledge contained in the group map, one participant questioned: "what knowledge are we not aware that are we missing? How do we find out?" This was an example of the general issue of being able to 'know what you don't know'.

The second session asked "What processes are used to acquire, share, retain and utilize knowledge?” Post-workshop, these processes were analysed on a 2x3 matrix to identify which were formal or informal and which were oral or written or electronic. A quantitative analysis 
is provided in Table 1. Nearly half of the existing processes within HighTechManuf were formal, written processes (for example, standard operations sheets, key performance indicators, reports and guidelines, quality records and drawings). Moreover, the participants thought that improving these written, formal processes was one of the best ways to improve their practice of knowledge management (participants wanted to improve 33 of the 51 processes currently used). Formal, oral processes were also felt to be a useful way of sharing knowledge, for example, weekly communication meetings. However, there was a significant amount of frustration in the participants about having too many meetings. This was seen as a barrier to knowledge management (due to information overload). Electronic formal processes included shared files on computer, databases and a single point of access to information.

\{Take in Table 1 “The types of knowledge to be managed”\}

In contrast there was felt to be a lack of informal, oral processes, and there was much debate about depending on "random bumpings" between people as a means of knowledge management. Comments included:

"They're not working if they're stood around the coffee machine", a reply to which was "we don't even have a coffee machine". However, one participant commented "I think we should encourage it - in a formal meeting you do not say what you might informally" while another observed that "It's not a process you can formalise"

Electronic informal processes were largely about "random email" and bulletin boards. Experiential processes included on the job training and apprenticeship. Other processes included using industry experts and consultants, competitor analysis via tradeshows.

After a vote, participants decided to depart from the original agenda, and concentrate on improving three specific aspects of knowledge management: the use of standard operating sheets; social networks; meetings.

The third session therefore asked the question "How do you get the information into standard operating sheets?” but the focus was wider, covering the motivation for sharing information and the use of standard operating sheets (SOPs). The group focussed on four issues. First “enablers” were identified, including: developing a common language across the three units; creating a generic methodology for their development; identifying skills which the company might be at risk of losing.

Second, methods for “capturing key information” were discussed, including: confiscating personal black books which cannot be shared; breaking up the jobs into small elements and 
agreeing and documenting in a consistent way the best (and worst) ways of doing those; using visual representations (photos, drawings etc) or video in addition to the written word. However, one participant said "there are things we do not have a best practice on \{to put into a SOP\}, because we do not know how to do them yet."

Third, participants discussed how they would provide support and encourage workers to put information into SOPs: asking "what's in it for me?” Methods included: aligning reward and recognition incentives to knowledge sharing; training; linking standardised work to skills matrix and educating workers as to the benefits of standardised procedures, including Team Leaders; appointing champions.

Fourth, evaluation of the SOPs was discussed. This included: monitoring rework/scrap of reengineered processes; audits, evaluate and modify operations and SOPs; address recurring non-conformance; create a database of SOPs which is updated. Overall, changing the design and use of SOPs was considered to require a change in process, as well as (and probably more difficult) a change in the perspective of the workers in terms of their attitude towards SOPs and how dedicated they were to successfully implementing them.

The fourth session considered the importance of social and professional networks in the knowledge management strategy of HighTechManuf. The discussion did not get much beyond identifying the enablers, which included: gaining executive sponsorship; providing a budget; rewarding the achievements of networks. Discussion also considered the type of activities - brainstorming events, newsletters, special interest groups, social events, guest speakers, team rivalry dimension, off-site events. One participant noted that there might be "more of a tangible benefit from an accountant and a purchasing guy talking than two purchasing guys from different divisions.”

In the fifth, and last, session participants discussed the when, and how, of meetings. This arose out of a sense of desperation among the participants of being invited to too many meetings, which were often unfocussed, too long, and attended by the wrong people. It was decided that meetings should be called when: all the necessary information is available; multidisciplinary input or debate is required; it is imperative to avoid misunderstanding and to ensure a consistent message is conveyed; more than two people are involved. In terms of how meetings should be more effective the group decided that meetings should be: planned, with an agenda circulated well in advance; chaired effectively; short, sharp and task oriented (with a time limit); able to produce minutes and a list of actions within 24 hours. 


\section{ManufIndProd}

ManufIndProd is a privately owned manufacturer of industrial products for engineering and construction, formed through a recent management buy-out. There had been an informal recognition of the importance of knowledge for years, but only recently had the new senior management team begun to formalise the knowledge management processes. They accepted a need for cultural change in the longstanding workforce. The client (the Managing Director and co-owner of ManufIndProd) outlined the organisation's problem as: where is the knowledge? how can we utilise it? where do we want to be? how do we get to where we want to be?

The 10 participants represented the range of the hierarchy - from the top management team right down to team leaders - however the majority were from the manufacturing side of the organisation. The participants agreed that "I think that knowledge management is an important issue in ManufIndProd" (average 1.70, where 1 = Strongly Agree and 5= Strongly Disagree).

The first question was "What knowledge informs ManufIndProd?" Responses are displayed in Figure 2. Seeing the finished map, one participant reflected "that looks just like the ISO9000 chart", quickly responded to by another participant, "Yes, but it took two hours, not 15 weeks”.

\{Take in Figure 2: “A map from the ManufIndProd workshop”\}

The second question was "What processes are used to acquire, share, retain and utilize knowledge?” Post-workshop analysis of the views was performed using the same framework as for HighTechManuf (results in Table 1). In ManufIndProd well over half of the processes were formal, written in nature. Many of these processes were concentrating on manufacturing and technical knowledge, for example: process specifications and sheets; technical reports; quality manual; and vendor rating. Within ManufIndProd there was a strong commitment to using notice-boards for sharing formal, written knowledge and monitoring progress. Also, there was a desire to monitor process control more, again in a formal, written way. Additional examples are available for each category of knowledge as for HighTechManuf, but will not be reproduced here, except to observe that written informal processes included 'post-it' notes.

There was some concern over the number of processes they wanted to improve: they thought that 52 out of the 86 processes currently used could be improved. However, they also realised 
that this desire to improve was a healthy reflection of the desire of the organisation to improve its business.

As with HighTechManuf, participants decided to refocus Session 3 in the original agenda to address "What processes should we be using to improve our process control?" This was to lead to a final discussion, "What measures should be used to evaluate the improvement in process control?”

The third session therefore focussed on improving process control. This improvement was thought possible through improved: use of statistical process control, capability studies and cause and effect diagram; process recording including plan-do-check-act, improving the accurate capturing of relevant data; discipline through doing things properly, such as, tooling, labelling, paperwork traceability, planning; training to ensure it is adequate. Also recommendations for improvements covered: work instructions - many of the views were similar to those for how HighTechManuf might improve SOPs; ergonomics, lighting, space utilisation; quality assurance including shelf life control, tighter sampling control, and control of incoming materials.

One participant noted that "the men will fill in the work study sheet to fit the procedure, but that won't be what they \{actually\} do", suggesting that capturing useful data through this might be problematic.

The fourth, and final, session asked the question "What measures should be used to evaluate the improvement in process control?” Measures included: output from the statistical process control; improved stock management, including reduced WIP and obsolete stock; machine down-time and retooling times increasing utilisation; improved productivity, including energy consumption. Other measures pertained to: quality of the customer service, service levels and satisfaction; quality of the product in terms of number of returns and complaints; meeting due dates for delivery. There were also: business benefits of profitability, increased orders and lower running costs; and employee benefits of reduced absenteeism and 'smile charts'. In ManufIndProd most of these measures were already being done to some degree, but the group felt that they could do a lot more on many of them, and that this work could start immediately. 


\section{Discussion}

\section{The structure of the knowledge maps}

Figures 1 and 2 represent the knowledge that participants from the two organisations thought important. Although their detailed content seems very different, the two maps are remarkably similar in structure. HighTechManuf (Figure 1) has a cluster of operational knowledge (labelled "manufacturing") on the lower left-hand side. The remainder of the left-hand side consists of knowledge related to the structure part of manufacturing strategy, with a central cluster ("learning and sharing") of knowledge relating to the infrastructure part of manufacturing strategy. The right-hand side of the map comprises knowledge relating to corporate strategy. For ManufIndProd (Figure 2) the operations knowledge is in a different place (centre right, labelled "manufacturing product"). However, it is again surrounded by the knowledge related to the structure part of manufacturing strategy, with the infrastructure knowledge at the top on the left hand side, and the corporate strategy knowledge along the bottom.

These stand in sharp contrast to Figure 3, which shows the corresponding map for a nonmanufacturing organisation that was involved in the provision of social housing. In this map it is almost impossible to distinguish operational knowledge from strategic knowledge, and this is typical of these maps for non-manufacturing organisations.

\{Take in Figure 3: “A map from the Housing workshop”\}

It should be noted in this discussion that these maps, capturing as they do the opinions expressed during a particular session, are not perfect. The two activities at the top left in Figure 2, "credit rating information" and "customer prospects" are not joined to the rest of the map and clearly misplaced. The same is true of "IT support knowledge" (bottom left). (A principle of the Journey Making approach is that the maps are not normally amended after the workshop.) Nevertheless, the overall similarity between Figures 1 and 2 is striking, especially when set against the difference between these and the maps from Session 1 for nonmanufacturing organisations.

\section{The importance of core processes}

Looking in slightly more detail, the importance of core processes is apparent. In Figure 1 (HighTechManuf), eight of the eleven clusters related entirely to knowledge about core processes and their management, including the whole of the left hand side of the map (manufacturing operations and strategy). The "market knowledge" cluster was also mainly 
core, with one or two elements of support processes, while the "general management of the business” cluster was mainly about support processes and their management.

ManufIndProd (Figure 2) started with a strong focus on the customer and their requirements. Around 16 clusters were identified, although some of these were just one item, and as already mentioned "customer prospects" and "IT support knowledge" seems to have been 'orphaned'. Seven of the clusters were entirely knowledge about core processes and their management, covering almost half the map, from the right-hand side. "External sources of technical knowledge" and "internal sources of knowledge" were also mainly about the management of core processes, while "external general knowledge" was mainly management of support processes. The three small clusters (168, 169 and 133) were management of support processes. The remaining three, "business direction", "people” and "competitor activity” were mainly knowledge about management of support processes, although some of the "People" cluster seemed to relate more specifically to knowledge about the support processes themselves.

As Figures 1 and 2 demonstrate, much of the knowledge mentioned by participants was knowledge about core processes and their management. This was also seen as being the most important, as the topics discussed in most detail in later sessions showed. HighTechManuf's main emphasis was manufacturing operations - on standard operating sheets (SOPs) for knowledge sharing. ManufIndProd's was on process control - a strategic infrastructure aspect with the discussion having strong links to operations and structure aspects such as product characteristics and design. Management of these core processes was also discussed at some length in the workshops, including strategies for encouraging employee ownership of processes. By contrast, support processes received relatively little attention, except for that of retention of skills. The formal oral processes discussed in HighTechManuf also tended to be support processes. Sales is also a support process, although whether ManufIndProd really used the term "sales" to mean marketing (which would be core) is not certain.

\section{Process and culture (perspective) change}

While it may be obvious that the implementation of a knowledge management initiative will involve process change in the organisation, it will also require some culture, or perspective, change throughout the organisation: the top-down initiative needs to be matched with bottomup commitment and enthusiasm (Edwards and Kidd, 2003b). What was evident in both cases was the need for a cultural change in the perspective of the entire organisation towards knowledge management. 
The appointment of a knowledge champion (which both had done: in ManufIndProd it was the Managing Director) is an acknowledgement of the importance of knowledge management from the top of the hierarchy. One of the corresponding acknowledgements needed from lower in the hierarchy is the diligent recording of process information and knowledge. Both workshops raised concerns that changing the way in which paperwork is requested is more than just a change in process; it perhaps requires a change in the perspective of the worker about the importance of the process. The manufacturing infrastructure needs to support the structure in a very deep sense. A significant incident occurred during a break between sessions in the ManufIndProd workshop. One of the shop floor workers (a team leader) asked to speak to the facilitator. He was not sure about raising some rather controversial issues relating to the workforce taking management initiatives seriously. Previously, when the company had been part of a much larger organisation run from a 'remote' head office, the workers' reaction to any new initiative was to carry on regardless, but adapt the paperwork to look as if the new system was being operated, as mentioned above. Now, with the new management buy-out, at one level the workforce realised that the management genuinely wanted change, and genuinely valued the workers' inputs as to what that change should be. However, at another level there was still the deep-seated feeling that it was all another fad and if they ignored it, it would go away. The team leader found it very hard to make this point to the Directors who were present, but was encouraged to do so, and this led to valuable progress.

The following sub-sections pursue other aspects of "top down" and "bottom up" commitment.

\section{Management for informal and formal knowledge}

Results of the type of knowledge to be shared (see Table 1) were consistent for both organisations. Comparing these to other, non-manufacturing organisations from the original study (which represented a breadth of UK organisations, public and private), we found that the manufacturing organisations had a similar balance on the management of formal and informal knowledge. This suggests that the task facing manufacturing organisations is not dissimilar to that for organisations in other sectors. Also, as for the other organisations, the participants' desire to improve the majority of these processes suggests a widespread desire for better knowledge management.

\section{Informal oral processes}

While informal (in particular, oral) knowledge sharing will always happen in organisations there was some disagreement in HighTechManuf and ManufIndProd over whether it should be encouraged. Briefly, the opposition to informal knowledge management processes felt that 
what might be shared is gossip, personal views or rumour which will be mistaken for fact, and that it cannot easily and consistently be shared around the wider organisation if it is not formal. The support for informal knowledge management processes felt that: enhanced personal relationships will make for more effective working; you will share some things in a more relaxed setting that you would not readily share in a meeting; sometimes you can have spontaneously innovative thoughts in a corridor conversation.

Davenport and Prusak (1998) promote the merits of informal knowledge management - they note the importance of the water-cooler as a focus for congregating. However, in Hill's (1987) terms, these difficulties are apparent, because of the confusion of structural and infrastructural aspects that may result.

\section{Taking ownership of information processes}

In both workshops, participants were keen to ensure that information and knowledge stay current through constant up-dating. HighTechManuf thought that this might be encouraged through individuals taking the ownership of processes which produce the information and knowledge. Both the knowledge management and business process literatures would support this. However, Collinson (1999) found approaches for similar types of empowerment to be “difficult, and change will take longer than anticipated" page 355. Again, this is a longerterm, infrastructural measure, when results may be needed in the shorter term.

A key element in such a change would be a role for a senior and respected manager as champion of knowledge management, actively promoting a collectivistic culture and encouraging the free sharing of knowledge and information. Glazer (1998) identifies some of the many benefits this can deliver.

\section{Communicating knowledge}

Participants in both organisations were frustrated with the lack of effective communication of knowledge. Complaints included: meetings were unfocussed and too long with the wrong people being present; email was abused by people sending indiscriminately; briefings were not of the right information. In most cases the solutions to these problems were straightforward, yet it was only through confronting the issue in a group that commitment to action began to be built. However, as few of these mechanisms involved core knowledge, it is possible that participants were subconsciously giving these matters a lower priority on their action list. 


\section{Action plans to improve knowledge management}

Despite the enthusiasm of the participants to make progress in knowledge management strategy, there was often an element of frustration and despair at the enormity of the task. To focus and co-ordinate the efforts of the organisation we encouraged each to develop action plans, including both quick-wins and longer-term actions (Shaw and Edwards, 2005 (to appear)). Quick-wins were designed to build momentum from realising the benefits from knowledge management, and to help build enthusiasm in rest of the organisation. Some of these quick-wins included: updating SOPs through tying a laminated SOP sheet and a marker pen to each machine so that the operator can update the SOP in line with current operations; issuing a protocol for deciding when a meeting should be called and who should be invited; improving team briefings.

Longer-term actions were those which would need significant resources to realise. These included: the design of training programmes to share process knowledge among the workforce; harnessing the full benefits from statistical process control; synthesising the use of integrated databases to break the "islands of databases" as in HighTechManuf.

\section{Using workshops for manufacturing knowledge management}

Questionnaire responses provide strong support from participants that the workshops were beneficial for their development of knowledge management strategy. Participants agreed: "I enjoyed the workshop” (average 1.65, where 1 = Strongly Agree and 5= Strongly Disagree), "the process was useful in helping us to explore knowledge management” (average 1.88) and agreed that they "had ability to share ideas with group members" (average 1.88). In terms of the action plans which were generated, participants agreed that: "I think that the outcome of the workshop was the right list of things that we need to do" (average 2.31); "I hope that these outcomes will influence what our organisation does on knowledge management.” (average 1.88); "I think that the outcome was generated in an appropriate way" (average 1.88); and "I feel that I have had an impact on the outcome” (average 2.13). All these are factors that are crucial for the participants' continued support of these actions through to their implementation (Eden and Ackermann, 1998).

Group workshops also have the benefit that misunderstandings can be identified and queried for example, the term "rates per hour" was assumed by operational staff to be output, yet a member of support staff interpreted it to be pay rate. 


\section{Limitations}

The most obvious limitations of this study are that it is based on only two organisations, and a limited number of participants from each. Concerns were expressed in the workshop about biased representation in the group. One ManufIndProd participant commented "a broader cross-section of staff may have produced a different set of priorities" as no-one from sales was present. In HighTechManuf the group focused on the issues which participants felt they could most usefully handle - there was a lack of people with responsibility for quality systems in the group, but the group only realised this as quality began to be discussed.

This underlines the importance of the selection of participants, but also that knowledge management permeates all functions of the organisation. Any implementable knowledge management strategy needs, at minimum, to include a wide range of functions and, we believe, to synthesise and be respectful to the competing priorities of these different functions.

\section{Conclusions}

Two manufacturing organisations concentrated on identifying and designing a strategy for better knowledge management. Both organisations, although relatively new to knowledge management as an explicit activity, found it easy and natural to concentrate on their core knowledge, much of it concerning the manufacturing process. These are areas where the link to bottom-line results is the clearest. Nevertheless, the knowledge maps show that knowledge management strategy cuts across operations, manufacturing strategy and corporate strategy. It should not be seen as a subset of either of the latter two.

In terms of implementing the knowledge management strategy, both top down and bottom up actions were needed. From the top, successful implementation might be supported through a knowledge champion promoting a collectivistic culture, implementing reward structures appropriate to such a culture, and the influence of line management in reinforcing this. The latter applies especially to informal methods of sharing knowledge. These may be effective, but some managers find it hard to allow them to take place. From the bottom up, the workforce needs to take ownership of processes, and to make sure that descriptions of the process remain in step with what actually happens. Training was viewed as an important means of sharing and retaining knowledge, but can only work if what is recorded matches what is done.

To be a knowledge-led company it seems crucial that all categories of knowledge are managed: within, across and between functions. Manufacturing organisations may have an 
advantage here in that it may be easier for them to distinguish between operational and strategic knowledge than it is for non-manufacturing organisations.

A fruitful area for future work could be to explore the consistency of knowledge management strategy with the organisation's manufacturing and corporate strategies.

As for the workshops, they seemed appropriate for enabling people from different manufacturing functions in the same organisation to share their knowledge about how to improve knowledge management processes. The workshops enabled the participants' negotiation on different action plans in pursuit of a strategy which was appropriate for implementation.

\section{Acknowledgement}

This research was funded by CIMA, the Chartered Institute of Management Accountants in the UK.

\section{References}

Beckett, A. J., Wainwright, C. E. R. and Bance, D. (2000), "Implementing an industrial continuous improvement system: A knowledge management case study", Industrial Management and Data Systems, Vol 100, No. 7, pp.330-338.

Bhatt, G. D. (2000), "Organizing knowledge in the knowledge development cycle", Journal of Knowledge Management, Vol 4, No. 1, pp.15-26.

Chaston, I., Badger, B., Mangles, T. and Sadler-Smith, E. (2001), "Organisational learning style, competencies and learning systems in small, UK manufacturing firms", International Journal of Operations \& Production Management, Vol 21, No. 11, pp.1417-1432.

Chaston, I. and Mangles, T. (2000), "Business networks: assisting knowledge management and competence acquisition within UK manufacturing firms", Journal of Small Business and Enterprise Development, Vol 7, No. 2, pp.160-170.

Collinson, S. (1999), "Knowledge management capabilities for steel makers: A BritishJapanese corporate alliance for organizational learning", Technology Analysis and Strategic Management, Vol 11, No. 3, pp.337-358.

Davenport, T. H. and Prusak, L. (1998), Working knowledge: how organizations manage what they know, Harvard Business School Press, Boston, Mass.

Earl, M. J. (1994), "The new and the old of business process redesign", The Journal of Strategic Information Systems, Vol 3, No. 1, pp.5-22.

Eden, C. and Ackermann, F. (1998), Making Strategy: The Journey of Strategic Management, Sage, London.

Edwards, J. S. and Kidd, J. B. (2003a), "Bridging the gap from the general to the specific by linking knowledge management to business processes", in Hlupic, V. (Ed.) Knowledge and Business Process Management, Idea Group, Hershey PA, pp.118136.

Edwards, J. S. and Kidd, J. B. (2003b), "Knowledge Management sans frontières", Journal of the Operational Research Society, Vol 54, No. 2, pp.130-139. 
Germain, R., Droge, C. and Christensen, W. (2001), "The mediating role of operations knowledge in the relationship of context with performance", Journal of Operations Management, Vol 19, 453-469.

Glazer, R. (1998), "Measuring the knower: Towards a theory of knowledge equity", California Management Review, Vol 40, No. 3, pp.175-194.

Hayes, R. H. and Wheelwright, S. C. (1984), Restoring our competitive edge, Collier Macmillan, New York.

Hill, T. J. (1987), "Teaching manufacturing strategy", International Journal of Operations \& Production Management, Vol 6, No. 3, pp.10-20.

Leseure, M. and Brookes, N. (2001), "Micro knowledge management: A job design framework", in Roy, R. (Ed.) Industrial knowledge management: A micro-level approach, Springer-Verlag, pp.163-178.

Minor, E. D., Hensley, R. L. and Wood, D. R. (1994), "A review of empirical manufacturing strategy studies", International Journal of Operations \& Production Management, Vol 14, No. 1, pp.5-25.

Misterek, S. D. A., Schroeder, R. G. and Bates, K. A. (1992), "The nature of the link between manufacturing strategy and organizational culture", in Voss, C. A. (Ed.) Manufacturing strategy: process and content, Chapman \& Hall, London, pp.331-352.

Mohanty, R. P. and Deshmukh, S. G. (1999), "Evaluating manufacturing strategy for a learning organization: A case", International Journal of Operations and Production Management, Vol 19, No. 3, pp.308-327.

Ordonez de Pablos, P. (2002), "Knowledge management and organizational learning: Typologies of knowledge sharing in the Spanish manufacturing industry from 1995 to 1999", Journal of Knowledge Management, Vol 6, No. 1, pp.52-62.

Paiva, E. L., Roth, A. V. and Fensterseifer, J. E. (2002), "Focusing information in manufacturing: A knowledge management perspective", Industrial Management and Data Systems, Vol 102, 381-389.

Platts, K. W. and Gregory, M. J. (1992), "A manufacturing audit approach to strategy formulation", in Voss, C. A. (Ed.) Manufacturing strategy: process and content, Chapman \& Hall, London, pp.29-55.

Prasad, B. (2000), "Converting computer-integrated manufacturing into an intelligent information system by combining CIM with concurrent engineering and knowledge management", Industrial Management and Data Systems, Vol 100, 301-316.

Robertson, M., Swan, J. and Newell, S. (1996), "The role of networks in the diffusion of technological innovation", Journal of Management Studies, Vol 33, No. 3, pp.333359.

Robinson, S., Edwards, J. S. and Yongfa, W. (2003), "Linking the Witness simulation software to an expert system to represent a decision-making process", Journal of Computing and Information Technology, Vol 11, No. 2, pp.123-133.

Shaw, D. and Edwards, J. S. (2005 (to appear)), "Building user commitment to implementing a knowledge management strategy", Information \& Management. 


\begin{tabular}{|c|c|c|c|c|c|c|c|c|}
\hline & \multicolumn{2}{|c|}{ HighTechManuf } & \multicolumn{2}{|c|}{ ManufIndProd } & \multicolumn{2}{|c|}{ Housing } & \multicolumn{2}{|c|}{$\begin{array}{c}\text { Average for other } \\
\text { organisations }\end{array}$} \\
\hline & Formal & Informal & Formal & Informal & Formal & Informal & Formal & Informal \\
\hline Oral & $17 \%$ & $10 \%$ & $9 \%$ & $1 \%$ & $25 \%$ & $6 \%$ & $18 \%$ & $10 \%$ \\
\hline Written & $46 \%$ & $0 \%$ & $62 \%$ & $1 \%$ & $49 \%$ & $2 \%$ & $49 \%$ & $1 \%$ \\
\hline Electronic & $8 \%$ & $6 \%$ & $4 \%$ & $1 \%$ & $4 \%$ & $6 \%$ & $10 \%$ & $3 \%$ \\
\hline Experiential & \multicolumn{2}{|c|}{$6 \%$} & \multicolumn{2}{|c|}{$7 \%$} & \multicolumn{2}{|c|}{$8 \%$} & \multicolumn{2}{|c|}{$3 \%$} \\
\hline Other & \multicolumn{2}{|c|}{$6 \%$} & \multicolumn{2}{|c|}{$13 \%$} & \multicolumn{2}{|c|}{$0 \%$} & \multicolumn{2}{|c|}{$6 \%$} \\
\hline
\end{tabular}

Table 1: The types of knowledge to be managed 


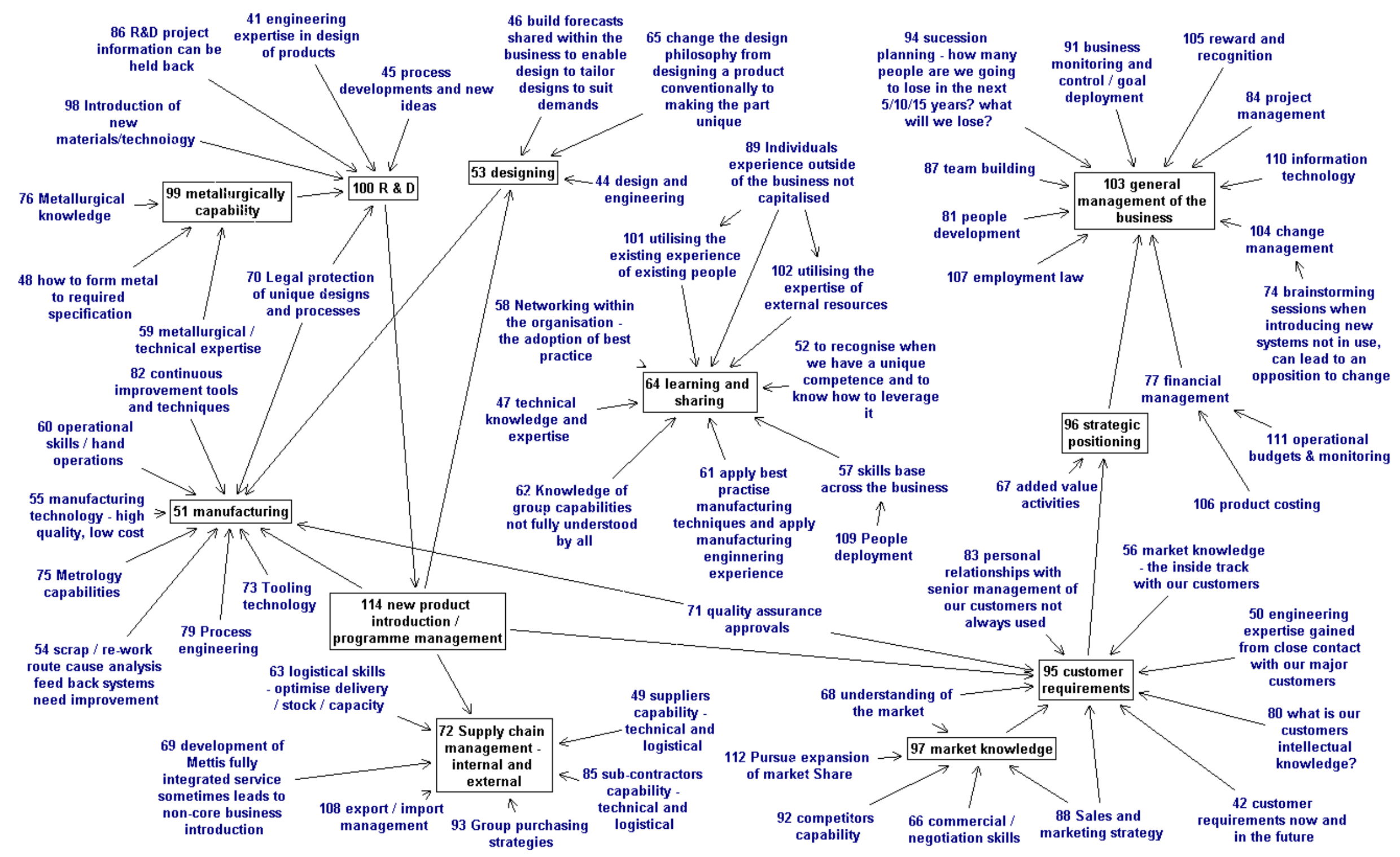

Figure 1: “A map from the HighTechManuf workshop” 


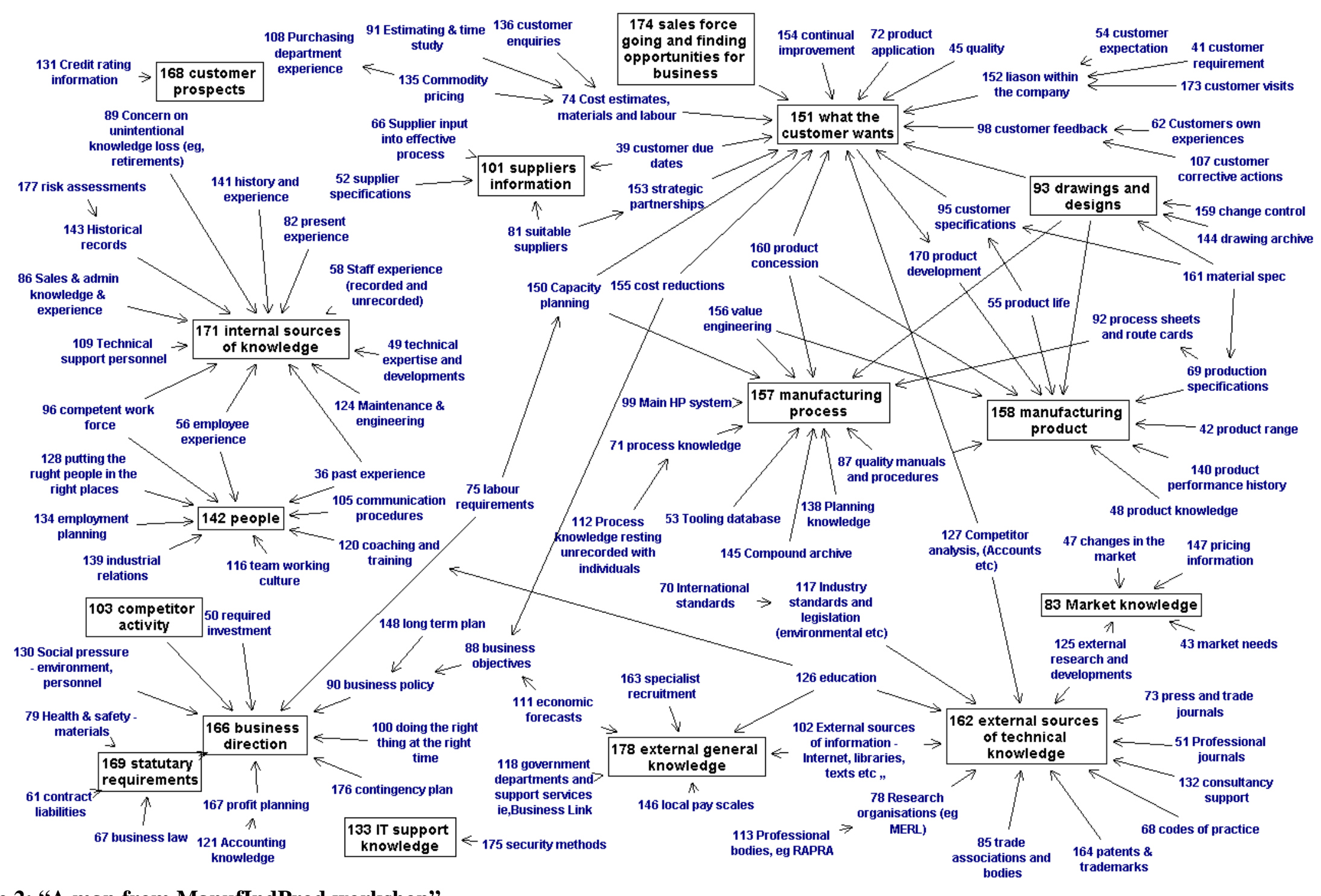

Page 22 


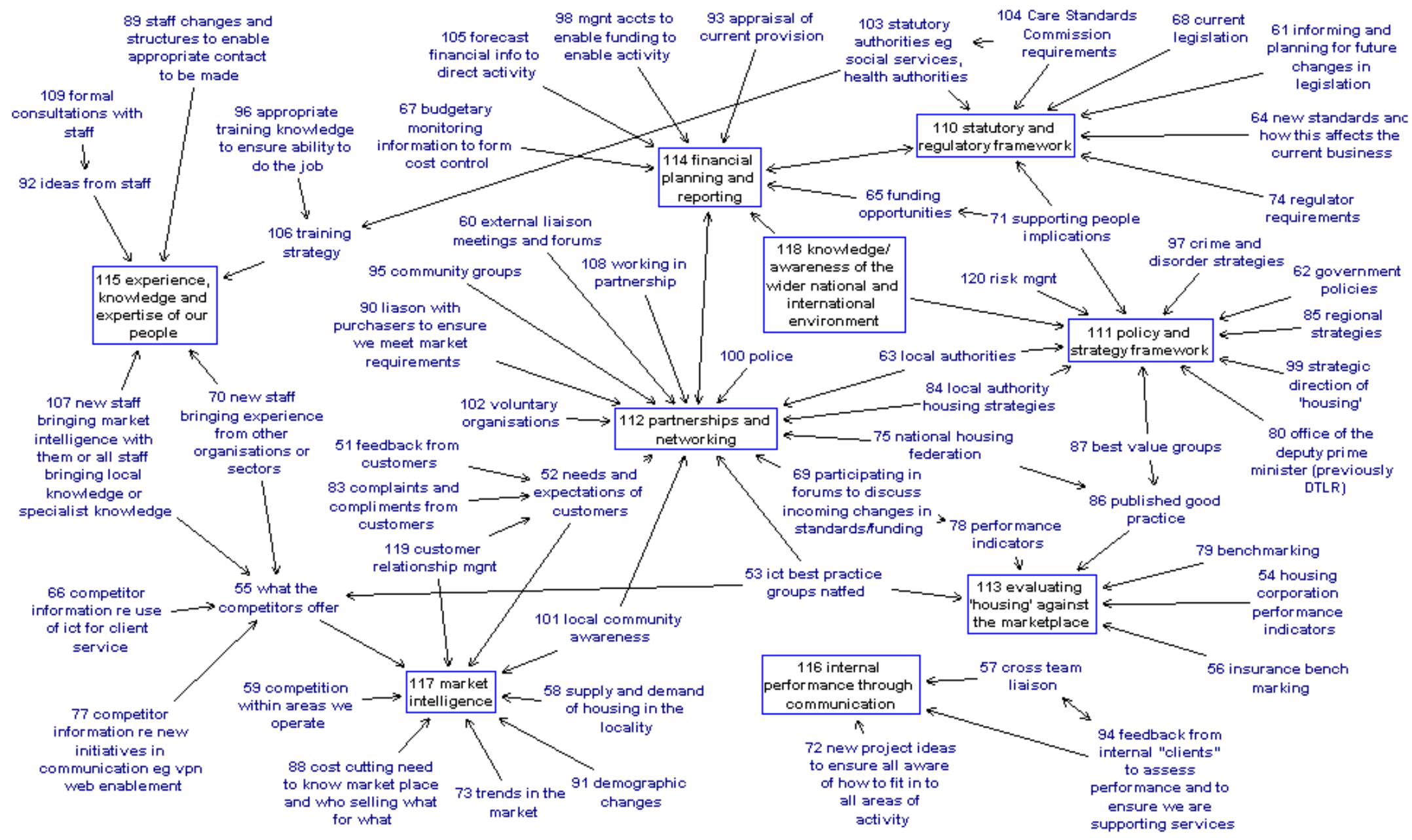

Figure 3: “A map from Housing workshop” 